\title{
Performance analysis of modified tent map interleaver in IDMA systems
}

\author{
Aasheesh Shukla, Vinay Kumar Deolia *
}

\begin{abstract}
In the last years, Interleave Division Multiple Access (IDMA) has been presented as a potential alternate of Code Division Multiple Access (CDMA) system. In IDMA systems, the interleavers are used to separate the users of the system in multiple access environments. Random interleaver is popular and basic taxonomy, which scrambles information bits of craving users with different patterns. However the indispensable characteristics of a random interleaver such as bandwidth requirement, computational complexity, and memory restraint at both transmitter and receiver end is uttermost. Further, it has also been observed that the study of role of chaos in interleaver design is very limited in literature. Hence, in this paper, a low complexity chaos based interleaver named as modified Tent map interleaver is designed for further performance improvement of IDMA system and the characteristic parameters are compared with the random interleaver. The IDMA system model uses a BPSK modulation and repetition coder with a code rate of $1 / 2$. The system is simulated in MATLAB and results show that the better BER performance without the need of extra memory resources.
\end{abstract}

K e y w or d s: IDMA, tent map, chaos, random interleaver, computational complexity

\section{Introduction}

It is widely known that interleave-division multiple access (IDMA) scheme is the promising candidate for future multiple access communication requirements that employs chip-level interleavers for user separation, unlike CDMA scheme where each user is assigned a spreading sequence. This technique overcomes the limitations of CDMA system such as multiple access interference (MAI), and intersymbol interference (ISI) [1-3]. Beside user separation, interleavers are also used to randomize the error bursts. Hence, an efficient interleaver can improve the performance of iterative IDMA system. However, the design of interleavers for multiple access (MA) systems is quite different from that of Turbo codes. For MA systems the interleavers should be easy to generate, non-colloidal in nature and consumed less bandwidth and memory resources $[4-6]$.

Recent research work has manifested the role of chaos in spread spectrum communication systems, working at the level of code design and random data generation can be more efficient. Moreover, chaotic sequences offer many interesting properties subjected to computational complexity or storage requirement [7-10]. More specifically, chaotic sequences can be used for interleaver design and yield a promising bit error rate performance in IDMA system. In this paper, a new chaotic map based interleaving design is being introduced named as "Modified Tent Map Interleaver" (MTMI) in IDMA scheme. The proposed interleaver has stronger randomness, relatively larger Lyapunov exponent, less computational complexity and memory requirement [11-14]

\section{System Model}

This is to note that the conventional code-division multiple access (CDMA) systems have some limitations and to mitigate them, IDMA system has been proposed. In IDMA system, users are separated by user specific chip-level interleavers. At the transmitter, the $n$-length input data sequence $b_{k}=\left[b_{k 1}, b_{k 2}, \ldots, b_{k i}, \ldots, b_{k n}\right]$ of $k$ th user $k \in(1,2,3, \ldots, N)$ is spreaded and encoded into chips $c_{k}=\left[c_{k 1}, c_{k 2}, \ldots, c_{k j}\right]$, where $j$ is the Chip length. The spreader and encoder are combined and belonging to code word $c$, which is same for all users. Next, chips are interleaved by a specific interleaving pattern (based on modified chaotic Tent map) to produce transmitted chip sequence $x_{n}$ [3]. The receiver for IDMA system of $k$ simultaneously transmitting users are comprised of multiuser detector (MUD) and posterior probability (APP) decoders in connection with user specific interleavers. The APP decoders and interleavers exchange the soft information and calculate the log likelihood ratios (LLRs) [10]. These LLRs should be independent from the users and hence called as extrinsic LLRs.

The MUD receives the a priori LLR sent from the decoder and can be defined as $L_{a}^{m}\left(c_{m, n, k}^{\prime}\right)=\frac{P\left\{c_{m, n, k}^{\prime}=+1\right\}}{P\left\{c_{m, n, k}^{\prime}=-1\right\}}$. With the knowledge of this a-priori LLR, the goal of the multi user detector is to compute the posterior LLRs, which can be represented as

$$
L^{m}\left(c_{m, n, k}^{\prime}\right)=\ln \frac{P\left(c_{m, n, k}^{\prime}=+1 / y_{n}\right)}{P\left(c_{m, n, k}^{\prime}=-1 / y_{n}\right)} .
$$

Department of Electronics and Communication Institute of Engineering and technology GLA University, India, 281406 , aasheesh.shukla@gla.ac.in 


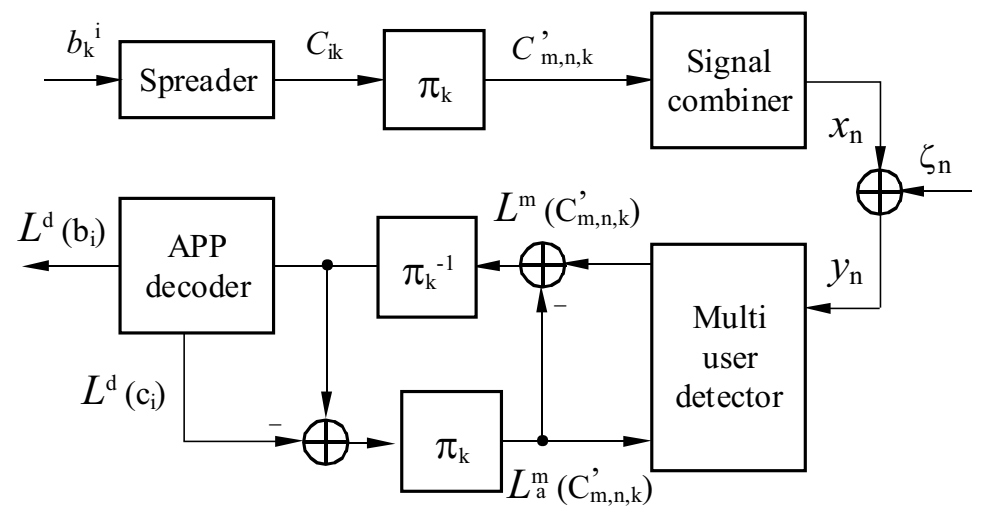

Fig. 1. - Transmitter and Receiver structure of IDMA for $k^{\text {th }}$ user

The extrinsic LLRs can be calculated as $L_{e}^{m}\left(c_{m, n, k}^{\prime}\right)=$ $L^{m}\left(c_{m, n, k}^{\prime}\right)-L_{a}^{m}\left(c_{m, n, k}^{\prime}\right)$ and then sent to the decoder after de-interleaving operation ie $\pi_{k}^{-1}$. Further, by using total probability theorem the extrinsic LLR can be written as $[2]$

$$
\begin{aligned}
& L_{e}^{m}\left(c_{m, n, k}^{\prime}\right)= \\
& \quad \ln \frac{\sum_{m, n, k}^{\prime}=+1}{\sum_{c_{m, n, k}^{\prime}=-1} P\left\{y_{n} \mid x_{n, k}=x\right\} \prod_{m^{\prime} \neq m} P\left\{c_{m, n, k}^{\prime}=x_{m^{\prime}, k}=x\right\} \prod_{m^{\prime} \neq m} P\left\{c_{m, n, k}^{\prime}=d_{m^{\prime}}\right\}} .
\end{aligned}
$$

Here $d_{m^{\prime}}$ denotes the $m$-th bit of symbol $x$. Each of decoder receives the de-interleaved extrinsic LLRs and channel LLRs $L_{c}^{d}$. Now with constraints on number of iterations, the decoder computed posterior LLRs $L^{d}$. To get extrinsic LLRs, the channel LLRs are subtracted from posterior LLRs ie $L_{e}^{d}=L^{d}-L_{c}^{d}$. After 5 to 10 iterations (to optimize delay), the decoder calculates posterior LLRs about the information bits $L^{d}\left(b_{i}\right)$. Taking the proper sign, it gives an estimate of information bits. Table 1 shows the list of all used notations and parameters.

Table 1. Used parameters

\begin{tabular}{lcc}
\hline S.no & Symbols & Description \\
\hline 1 & $b_{i, k}$ & Information bit for user $k$ \\
2 & $c_{j, k}$ & Coded bits \\
3 & $c_{m, n, k}$ & Interleaver output \\
4 & $x_{n}$ & Transmitted sequence \\
5 & $\xi_{n}$ & Noise vector \\
6 & $L^{m}\left(c_{m, n, k}^{\prime}\right)$ & Extrinsic LLR \\
7 & $L_{e}^{d}$ & Channel LLR \\
\hline
\end{tabular}

\section{Proposed modified tent map interleaver}

In the parameterized form, tent map, can be described piecewise by

$$
F_{m}(x)= \begin{cases}m x_{n}, & 0 \leq x \leq \frac{1}{2} \\ m\left(1-x_{n}\right), & \frac{1}{2}<\leq x \leq 1\end{cases}
$$

where $x_{0}$ is the initial state and $x \in[0,1]$ and parameter $m$ is known as bifurcation parameter. This parameter decides the qualitative behaviour of system and produce chaotic region for the values of $m \in(1,2]$. To increase the randomness of classical tent map, the improved and modified tent map has been suggested [12] and can be written as

$$
F_{m}(x)= \begin{cases}\left(x_{n}-a\left\lfloor x_{n} / a\right\rfloor\right) / a, & \forall\left\lfloor x_{n} / a\right\rfloor \text { even, } \\ \left(a\left(\left\lfloor x_{n} / a\right\rfloor+1\right)-x_{n}\right) / a, & \forall\left\lfloor x_{n} / a\right\rfloor \text { odd }\end{cases}
$$

where $x_{n} \in(0,1)$ and system parameter is taken as $a \in(0,0.5)$. The state values of improved Tent map shows larger randomness [12], so the interleaver based on modified Tent map provides better BER performance in IDMA system. Further, modified Map has larger values of Lyapunov exponents (LE), which implies that system (4) has better chaotic behaviour than classical Tent map. $C_{0}$ Complexity analysis is used to measure the complexity of chaotic system. Larger values of $C_{0}$ means for high complexity of system. The value of complexity for classical Tent map and improved map is given in Table 2 .

Table 2. $C_{0}$ complexity values for different system parameters

\begin{tabular}{ccc}
\hline $\begin{array}{c}\text { System } \\
\text { Parameter }(\mathrm{a})\end{array}$ & $\begin{array}{c}\text { Tent Map } \\
\left(C_{0} \text { values }\right)\end{array}$ & $\begin{array}{c}\text { Modified Tent Map } \\
\left(C_{0} \text { Values }\right)(\text { approx. })\end{array}$ \\
\hline 0.1 & 0.0012 & 0.252 \\
0.2 & 0.0012 & 0.254 \\
0.3 & 0.0012 & 0.223 \\
0.4 & 0.0012 & 0.209 \\
0.5 & 0.0012 & 0.262 \\
\hline
\end{tabular}

For a wider range of system parameters the Modified Tent map provides different values of $C_{0}$ and hence more complex and chaotic than classical Tent map. So the MTMI system is more suitable for application in secure communication and cryptography [4]. The algorithm for proposed modified tent map interleaver shows the method of generation of interleavers. 


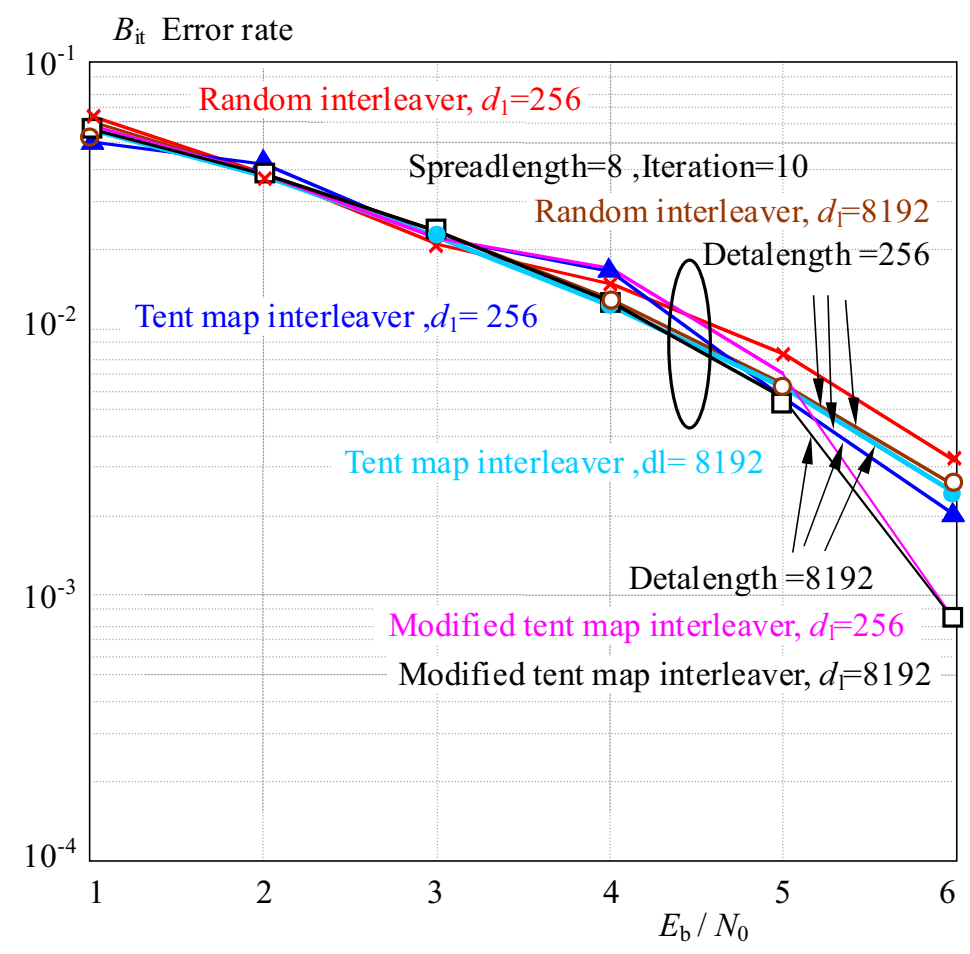

Fig. 2. The BER performance of modified tent map interleaver for data lengths 512,8192 , spread lengths $=8$, iteration $=10$

\section{Algorithm}

1. Initialization: $m \in(1,2], N=$ Interleaver length, $a=0.4$,

2. $X_{j}^{k}=k^{\text {th }}$ user: $0<X_{j}^{k}<N, \xi=$ Foot step,

3. $F_{0}^{k}=\left|X_{0}^{k}\right|$ : the first element $\left(\Pi^{k}=F_{0}^{k}\right), j=0$ and $n=0$,

Main operation:

4. If $\lfloor x / a\rfloor=$ even

5. Calculate $X_{j+1}^{k}$,

6. $F_{j+1}^{k}=\left|X_{j+1}^{k}\right|$,

7. Now check if $F_{j+1}^{k}$ is in the set $\Pi^{k}$,

8. Increment $j$ by 1 and repeat the main operation,

9. Otherwise $\Pi^{k}=\Pi^{k} \cup F_{j+1}^{k}$,

10. If floor $\lfloor x / a\rfloor=$ odd

11. $F_{j+1}^{k}=\left|X_{j+1}^{k}\right|$,

12. $\Pi^{k}=\Pi^{k} \cup\left|X_{j+1}^{k}\right|$,

13. End.

Finally redundant bits are removed to find the interleaver vector. For the second user, the initial value or first value is modified by foot step $\xi$ and then used as initial state to generate the whole sequence. The whole operation is repeated to get second interleaver and so on.

\section{Error-rate analysis}

The performance of proposed algorithm is vindicated by the simulation model in MATLAB. Random Interleaver (RI) Tent map interleaver (TMI) and modified
Tent map interleaver (MTMI) are considered for bit error rate (BER) analysis of conventional IDMA system. For simplicity, BPSK modulation is considered in multipath Rayleigh fading channel. The data length for each user is assumed 512 and 8192 bits and spreading code for all users is simply a repetition code with the length $S=8$. Simulation results authenticate the performance of TMI in IDMA system. This is clear in Fig. 2 that the proposed modified Tent map interleaver (MTMI) achieves similar BER performance compared to RI and better for $E_{b} / N_{0} \geq 5$. Hence, modified Tent map algorithm can be more efficient for higher values of $E_{b} / N_{0}$.

\section{Computational complexity}

Other than BER analysis, Computational complexity is also a crucial parameter to find an efficient interleaver. Here, complexity measure is meant by the number of cycles required for the generation of spreading codes and interleaver matrix in terms of users. In this section, computational complexity is calculated for modified tent map interleaver and compare with the random interleaver and Tent map interleaver. Table 3 shows that the required number of cycles increases with the number of user $\mathrm{k}$ for random interleaver. It means that complexity of RI is dependent on users. However, the proposed design of MTMI is independent from the number of users. Computational complexity is presented in Table 3 and verified that computational complexity of modified tent map interleaver is least possible[15-16]. 
Table 3. Number of cycles requited for the generation of interleaver

\begin{tabular}{cccc}
\hline No. of Users & Random Interleaver & TMI & MTMI \\
\hline 1 & 1 & 1 & 1 \\
2 & 2 & 1 & 1 \\
4 & 4 & 1 & 1 \\
16 & 16 & 1 & 1 \\
50 & 50 & 1 & 1 \\
\hline
\end{tabular}

\section{Memory requirement}

To decode or de-interleave the information bits, interleaving pattern must be known to the receiver. So, the key pattern is also sent along with transmitted data sequence, which leads the requirement of memory to store the interleaving pattern at the receiver end. The large amount of memory is required for large no. of users and becomes a major concern in the designing of interleaver for IDMA system. The proposed modified Tent map based interleaver algorithm suggests the need of only initialization parameter to generate a specific interleaver. Hence only single bit $i e$ initialization parameter is required to share between transmitter (MS) and receiver (BS). So, the modified TMI is efficient in memory requirement. In Table 4, memory requirement for different interleaver is presented. The spreaded data is represented as chip-length $\mathrm{cl}$ and $k$ is no. of users.

Table 4. Memory requirement for interleavers

\begin{tabular}{lc}
\hline Interleaver & $\begin{array}{c}\text { Memory Requirement } \\
\text { (chips/user) }\end{array}$ \\
\hline Random Interleaver & $k \times c l \times \log _{2}(c l)$ \\
Tent map Interleaver & $c l \times \log _{2}(c l)$ \\
Modified Tent map Interleaver & $c l \times \log _{2}(c l)$ \\
\hline
\end{tabular}

\section{Conclusion}

The main contribution of this paper is to propose the new design of modified TENT map interleaver, based on the chaos theory. It achieves good bit error rate performance as well as it is potentially less complex computationally. The simulation results verify that the storage requirement in modified TMI is independent of users. This improved technique is also compared with Random and Tent map interleaver and the superiority is validated through simulation results.

\section{REFERENCES}

[1] T. Yoshida, H. Mori and H. Shigematsu, "Analytic Study of Chaos of the Tent Map: Band Structures, Power Spectra, and
Critical Behaviours", Journal of Statistical Physics vol. 31, no. 2, 1983 pp. 279-308.

[2] K. Katsutoshi, G. Bauch and W. Utschick, "IDMA vs CDMA: Analysis and Comparison of Two Multiple Access Schemes", IEEE Transactions on wireless communications vol. 11, no. 1, 2012, pp. 78-87.

[3] B. Akbil and G. Ferre, "The NLM Interleaver Design for IDMA system", Proc of IEEE international conference on complex system, 2012, pp. 1-5.

[4] L. Shan, H. Qiang, J. Li and Z. Wang, "Chaotic Optimization Algorithm based on Tent Map", Control and Decision vol. 20, no. 2, 2005, pp. 179-182.

[5] O. M. Kwon, J. H. Park and S. M. Lee, "Secure Communication based on Chaotic Synchronization via Interval Time-Varying Delay Feedback Control", Nonlinear Dynamics vol. 63, no. 2, 2011, pp. 239-252.

[6] Y. Cao, "A New Hybrid Chaotic Map and its Application on Image Encryption and Hiding", Mathematical Problems Engineering, 2013, Article ID 728375.

[7] M. J. Ostami, S. Saryazdi, H. Shahba and A. Nezamabadi-Pour, "Chaos-Based Image Encryption Using Sum Operation Modulo 4 and 256", IETE Journal of Research vol. 62, no. 2, 2016, pp. 179-188.

[8] M. Eisencraft, R. D. Fanganiello, J. M. V. Grzybowski, D. C. Soriano, R. Attux, A. M. Batista, E. E. N. Macau, L. H. A. Monteiro, J. M. T. Romano, R. Suyama and T. Yoneyama, "Chaos-based Communication Systems Non-Ideal Channels", Communications Nonlinear Science and Numerical Simulation vol. 17 , no. 12 , 2012, pp. $4707-4718$.

[9] F. J. Escribano, A. Wagemakers and M. A. F. Sanjuan, "ChaosBased Turbo Systems Fading Channels", IEEE Transaction on circuits and systems vol. 61, no. 2, 2014, pp. 530-541.

10] L. Ping, L. Liu, K. Wu and W. K. Leung, "Interleave-Division Multiple-Access", IEEE Trans.on Wireless Comm. vol 5, no. 4, 2006, pp. 938-947.

[11] M. Sangeetha and V. Bhaskar, "PN Codes Versus Chaotic Codes: Performance Comparison a Gaussian Approximated Wideband CDMA System over Weibull Fading Channels", Journal of the Franklin Institute vol. 351, no. 6, 2014, pp. 3378-3404.

[12] X. Liao, "Improved Tent Map and Its Applications Image Encryption", International Journal of Security and Its Applications vol. 9, no. 1, 2015, pp. 25-34.

[13] I. Pupeza, A. Kavcic and Li Ping, "Efficient Generation of Interleavers for IDMA", Proc of IEEE International Conference on Communications, 2006, pp. 1508-1513.

[14] M. Shukla, V. K. Srivastava and S. Tiwari, "Analysis and Design of Optimum Interleaver for Iterative Receivers IDMA Scheme", Wireless Communications and Mobile Computing vol. 9, no. 10, 2009, pp. 1312-1317.

Received 19 April 2017

Aasheesh Shukla obtained his Master degree in Electronics and Communication Engineering from HBTI, Kanpur. He is currently pursuing $\mathrm{PhD}$ in GLA University, Mathura. His areas of interest include Multiple Access schemes, IDMA and other latest trends in technology. He has more than 10 years of experience in teaching and research in the area of wireless communication.

Vinay Kumar Deolia is working as a Professor and Head of the Department of Electronics and Communication Engineering, Institute of Engineering and Technology, GLA University, Mathura, India. He completed his $\mathrm{PhD}$ in Backstepping control from NIT, Allahabad, India. His areas of interest include non linear control systems, IDMA, and other latest trends in technology. He has more than 20 years of experience in teaching and research. 


\title{
Run length limited error control codes construction based on one control matrix property
}

\author{
Peter Farkaš, ${ }^{*}$ Frank Schindler ${ }^{* *}$
}

\begin{abstract}
In this manuscript a simple method is presented for constructing run length limited error control codes from linear binary block codes. The run length limited properties are obtained via addition of a carefully chosen fixed binary vector - a modifier to all codewords without introducing any additional redundancy. Modifier selection is based on a specific property, which can be found in some of the linear binary block codes control matrices. Similar known methods are based on properties of generator matrices. However some codes are specified via control matrices, for example low density parity check codes. The method proposed in this letter could be applied to some of them directly. This is illustrated in this manuscript using example in which a run length limited low density parity check code is obtained from Gallager code.
\end{abstract}

K e y w or d s: run length limited error control codes, control matrix, linear block codes, modifier

\section{Introduction}

A run-length limited error control code (RLL-ECC) has constrained run-lengths of identical symbols and can be used for error control. The research of RLL-ECC can be traced back to the late eighties of the 20-th century [12]. In [3] a new modified construction was presented yielding improved RLL properties for DC free error-correcting codes. In [4] trellis RLL-ECCs were constructed. In [5] a RLL-ECC for one error correction was proposed. In [6] a systematic approach was given for obtaining RLL-ECCs from binary error-correcting codes. The storage system industry motivated research into relatively complex RLLECCs based on turbo codes and low density parity check (LDPC) codes [7-11]. Recently, new RLL Codes were proposed for visible light communication (VLC) systems together with soft decoding methods [12-14]. In [15] algorithms were presented for code design using finite state machines which simultaneously provide a coding gain while also mitigating flicker in VLC. In [16-23] RLLECCs were constructed using the arrangement illustrated in Fig. 1, from linear binary block codes (LBBC) using modifiers obtained based on generator matrix properties. This method has a disadvantage, that it cannot be used directly for codes that are specified via control matrices, for example LDPC codes. In contrast to this method, the approach proposed in this letter could be applied to some of them directly because the modifiers are selected using a specific property, which can be found in some LBBC control matrices.

\section{Basic properties of Linear Binary Block Codes Matrices}

If $C$ is an $k$-dimensional LBBC in which each codeword $\mathbf{c}$ has length $n$, it could be defined using a $[k \times n]$ generator matrix over a finite field $G F(2)$. The control matrix for $C$ is defined in [24] as

$$
C=\left\{\mathbf{c} ; \mathbf{c H}^{\top}=\mathbf{0}\right\} .
$$

Each row of $\mathbf{H}$ describes one control equation, which each codeword must fulfil. For example the following $\mathbf{H}$ matrix

$$
\mathbf{H}=\left[\begin{array}{lllllll}
0 & 0 & 0 & 1 & 1 & 1 & 1 \\
0 & 1 & 1 & 0 & 0 & 1 & 1 \\
1 & 0 & 1 & 0 & 1 & 0 & 1
\end{array}\right],
$$

which describes a $[7,4,3]$ Hamming code, defines for each codeword $\mathbf{c}=\left(c_{6}, c_{5}, c_{4}, c_{3}, c_{2}, c_{1}, c_{0}\right)$ following 3 control equations

$$
\begin{aligned}
& c_{3}+c_{2}+c_{1}+c_{0}=0, \\
& c_{6}+c_{5}+c_{1}+c_{0}=0, \\
& c_{6}+c_{4}+c_{2}+c_{0}=0 .
\end{aligned}
$$

N o t e . Each LBBC contains one all zero codeword and therefore an infinite run of zeros could occur in an infinite sequence of codewords.

\section{Description of the proposed construction method}

In practice it is often necessary to avoid long runs of identical symbols in encoded sequences. The practical

* Faculty of Electrical Engineering and Information Technology, Slovak University of Technology, Ilkovičova 381219 Bratislava, Slovakia, p.farkas@ieee.org, ** Faculty of Informatics, Pan-European University, Tematínska 10, 85105 Bratislava, Slovakia 